\title{
An evaluation of the diagnostic efficacy of fine needle aspiration biopsy in patients operated for a thyroid nodular goiter
}

This article was published in the following Dove Press journal:

OncoTargets and Therapy

22 September 2016

Number of times this article has been viewed

\section{Dariusz Janczak ${ }^{\prime, 2}$ \\ Wiktor Pawlowski' \\ Tadeusz Dorobisz',3 \\ Dawid Janczak ${ }^{4}$ \\ Karolina Dorobisz ${ }^{5}$ \\ Michal Leśniak' \\ Agnieszka Ziomek' \\ Mariusz Chabowski ${ }^{1,2}$ \\ 'Department of Surgery, 4th Military Teaching Hospital, ${ }^{2}$ Department of \\ Clinical Nursing, Division of Nursing in Surgical Procedures, ${ }^{3}$ Department of Clinical Basics of Physiotherapy, ${ }^{4}$ Department of Palliative Care Nursing, Faculty of Health Science, ${ }^{5}$ Department of Otolaryngology, Head and Neck Surgery, Wroclaw Medical University, Wroclaw, Poland}

Correspondence: Mariusz Chabowski Department of Surgery, 4th Military Teaching Hospital, 5 Weigla Street,

50-98I Wroclaw, Poland

$\mathrm{Tel}+48261660247$

Fax +48261660245

Email mariusz.chabowski@gmail.com
Background: Thyroid cancer (TC) comprises 1\% of all carcinomas and is the most common malignancy of the endocrine system. The disease is more common in women, with its peak morbidity observed in 40-50-year-old patients. The main risk factors include radiation, iodine deficiency, hereditary background, and genetic mutations. Among all diagnosed thyroid nodules, $5 \%-30 \%$ will evolve into cancer. The gold-standard procedure in the preoperative evaluation of a nodular goiter, apart from ultrasonography, is fine needle aspiration (FNA) biopsy. The FNA biopsy is favored for its simplicity, safety, and high specificity and sensitivity rates.

Aim: The aim of our study was to evaluate the clinical efficacy of FNA based on the patients' register.

Materials and methods: In the Department of Surgery at the 4th Military Teaching Hospital in Wroclaw, 2,133 patients underwent thyroid surgery for thyroid goiter between 1996 and 2015. One hundred and eight cases of TC were diagnosed and of these, 66 patients had a preoperative FNA.

Results: Fourteen FNA biopsies (21\%) revealed cancer, all of which were confirmed in the postoperative histopathology, although six cases of FNA-diagnosed cancer revealed a different histological type postoperatively. Eighteen FNA biopsies (27\%) were suspected of being malignant. A disturbingly high rate of "benign" FNA biopsies (32 cases; 48\%) revealed TC after surgery.

Conclusion: It is of great importance that the quality and quantity of FNA biopsies that are performed have been improved, especially due to the wide adoption of the Bethesda cytological evaluation system. FNA biopsy remains an obligatory and valuable diagnostic tool in thyroid nodules, but it is still insufficient as a standard procedure. A preoperative biopsy should always be related to all the available clinical data in order to provide the best treatment option for each patient individually.

Keywords: thyroid cancer, fine needle aspiration biopsy, goiter, The Bethesda System for Reporting Thyroid Cytopathology (TBSRTC)

\section{Introduction}

Thyroid cancer (TC) is the most common malignancy of the endocrine system, as it constitutes $1 \%$ of all carcinomas. A significant increase in the incidence of TC has been observed over the last few years, mainly associated with diagnosis of papillary carcinoma. This tendency is thought to be a result of the wider utilization of preoperative diagnostic procedures such as ultrasonography and fine needle aspiration (FNA) biopsy, and therefore is rather an increase in the number of diagnosed cases than an actual higher level of morbidity. Over 1,700 patients develop TC in Poland every year. 
TC is divided into: 1) well-differentiated carcinomas papillary (most common, $>80 \%$ cases) and follicular, both of which encompass approximately $90 \%$ of all TCs and 2) poorly differentiated carcinomas - comprising medullary and anaplastic carcinomas. Medullary carcinomas can be hereditary, related to RET gene mutation, and/or associated with multiple endocrine neoplasia. Anaplastic cancer is the most aggressive in growth and has the lowest survival rate, as it often leads to suffocation within a few months of the diagnosis. The rarest TCs include various lymphomas, sarcomas, and squamous cell neoplasms. ${ }^{1-5}$

The mean age for TC diagnosis is 50 years. Women (W) are diagnosed with TC approximately five times more often than men (M), but the male sex is associated with a worse clinical outcome. Risk factors of TC are: radiation exposure, iodine deficiency, hereditary background, and genetic mutations. The gold-standard procedure for thyroid nodule evaluation, approved by the American Thyroid Association and subsequently by Polish associations, is an ultrasound-guided FNA biopsy. In 2008, the Bethesda system was established in order to provide clear and unified cytological specimen assessment criteria. ${ }^{6-8}$ It was recommended in Poland in 2010.

The aim of our study was to evaluate the clinical efficacy of FNA based on the patients' register. Over 100 patients undergo thyroid surgery annually in our Department of Surgery.

\section{Materials and methods}

This retrospective study involved patients diagnosed with multi-, single- or retrosternal nodular goiter, including Graves-Basedow disease. Data were collected from case histories along with postoperative specimen histopathology results and preoperative FNA biopsies. Patients were referred for surgery after a set of laboratory and imaging tests, FNA, and ear-nose-throat assessment. The following surgery extent pattern was developed in our department: single nodule - lobectomy and isthmusectomy; multinodular goiter - total thyroidectomy; preoperative TC diagnosis - total thyroidectomy and central neck dissection. One hundred and eight patients who had been operated on in the period 1996-2015 and who had a postoperative diagnosis of TC were enrolled in the study. In total 2,133 patients were referred for thyroid surgery during this period. All the patients provided written informed consent to have their data used in this study. This study was approved by the Lodz Medical University Ethics Committee.

\section{Results}

The overall incidence of TC in the study period was $5.06 \%$. Papillary (72 female vs 9 male patients) and follicular TCs (ten female vs three male patients) were mostly diagnosed. Less common findings included: six cases of medullary cancer (two in $\mathrm{W}$, four in $\mathrm{M}$ ), three cases of anaplastic cancer (two in $\mathrm{W}$, one in $\mathrm{M}$ ), and two cases of squamous cell TC in $\mathrm{W}$. Furthermore, one case of fibrosarcoma and two metastases from head and neck cancers were diagnosed - all in female patients. The mean age at diagnosis was: 1) papillary cancer, 49 years for $M$ and 51 years for $W$; 2) follicular cancer, 44 years $(\mathrm{M})$ and 53 years (W); 3) medullary cancer, 53 years (M) and 62 years (W); 4) anaplastic cancer, 80 years (M) and 65 years (W); and 5) squamous cell cancer, 31 years (W) (Table 1). An increase in the number of diagnosed papillary cancer cases was also observed: in the period 1996-2005, there were approximately two cases per year, while in the period 2006-2015, this had increased to almost 5.5 cases per year (Figure 1). The mean size of the thyroid nodules evaluated by ultrasonography was $24.4 \mathrm{~mm}$ for right lobe nodules and $23.6 \mathrm{~mm}$ for left lobe nodules. Microcalcifications were found in nine cases of right lobe nodules, six cases of left lobe nodules, and in one case bilaterally.

Sixty-six out of the 108 patients diagnosed with cancer postoperatively presented their FNA result on admission. These biopsies had been performed either in our hospital or in other academic or community centers. As the Bethesda system was not used before 2010, we decided to divide the FNA biopsies that we had collected into four descriptive groups: 1) FNA biopsies in which cancer was diagnosed, 2) FNA biopsies where there was a suspicion of malignancy or which were indeterminate, 3 ) benign FNA biopsies, and 4) nondiagnostic FNA biopsies.

Table I Sex and age distribution of different types of thyroid cancer in the period 1996-20I5

\begin{tabular}{llll}
\hline Type of thyroid cancer & Female sex and mean age & Male sex and mean age & Total (N=I08 pts) \\
\hline Papillary & 72 pts and 51 years & 9 pts and 49 years & 81 pts \\
Follicular & 10 pts and 53 years & 3 pts and 44 years & 13 pts \\
Medullary & 2 pts and 62 years & 4 pts and 53 years & 6 pts \\
Anaplastic & 2 pts and 65 years & 1 pt and 80 years & 3 pts \\
Other (squamous, & 5 pts & 0 pts & 5 pts \\
fibrosarcoma, metastasis) & & & \\
\hline
\end{tabular}

Abbreviation: pts, patients. 


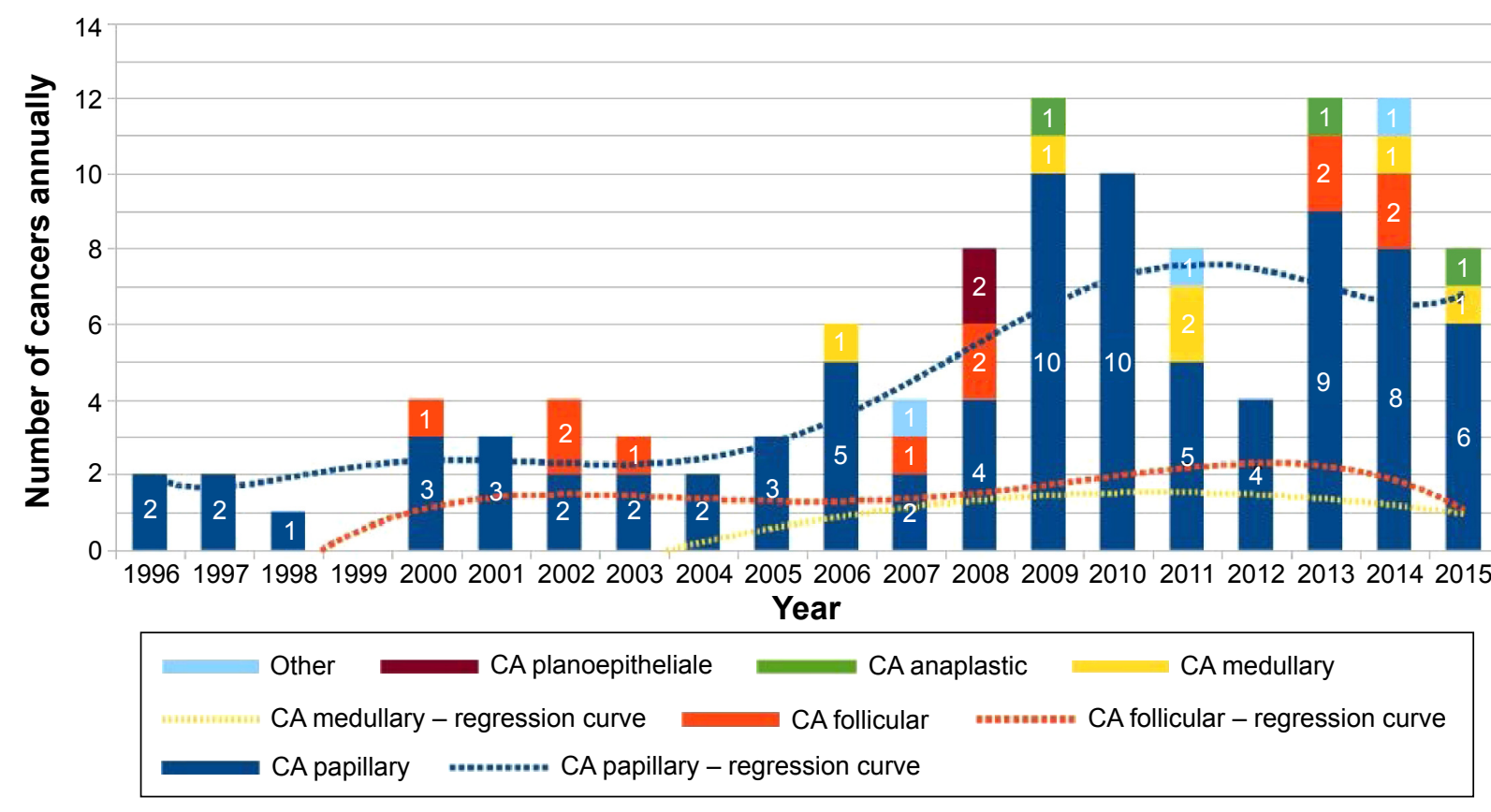

Figure I Thyroid cancer in postoperative specimen histopathology: 1996-2015. Abbreviation: CA, cancer.

TC was diagnosed in 14 of the FNA biopsies (21\%), all of which were confirmed in the postoperative specimen histopathology. However, in six of these cases, the postoperative diagnosis revealed a different type of TC than the initial FNA result. Two papillary cancers in FNA were reclassified as follicular and medullary cancer after surgery. Four cases of follicular cancer in FNA were classified as papillary cancers postoperatively. Eighteen FNA biopsies (27\%) indicated a suspicion of malignancy. A disturbingly high rate of false-negative (benign) FNA results was reported, that is, 32 patients (48\%), all of whom were diagnosed with TC on the final pathology after surgery. Two FNA biopsies were defined as nondiagnostic (Table 2). Thorough FNA analysis was difficult to perform in this study because the biopsy descriptions were of frequently low quality, which were, in some cases, limited to a few words. In recent years, a significant improvement in the quality of FNA biopsies has been observed, although only 15 biopsies out of the 31 obtained after 2010 referred to the Bethesda system.

Table 2 Results of the fine needle aspiration biopsy
Total 108 pts

Suspicion of malignancy

Benign - false negative

Nondiagnostic
Thyroid cancer

FNA not done - 42 pts (49\%)
FNA done -66 pts $(61 \%)$

14 pts $(21 \%)$ - including six pts of different type

18 pts $(27 \%)$

32 pts $(48 \%)$

2 pts $(3 \%)$
Abbreviations: FNA, fine needle aspiration; pts, patients.
In four patients, the postoperatively diagnosed $\mathrm{TC}$ was located in the contralateral lobe to that which it was expected on the basis of ultrasonography and FNA before surgery. One patient with preoperatively evaluated single-lobe thyroid nodules was diagnosed with bilateral TC on final histopathology after surgery. Two out of the five patients mentioned required further thyroid surgery in order to extend the range of thyroidectomy because of uncertainty about the surgical margins.

\section{Discussion}

Thyroid nodules are found in $4 \%-10 \%$ of the adult population. The overall risk of malignancy in a diagnosed nodule is $5 \%-30 \%$, irrespective of the initial diagnostic method. ${ }^{2,9-13}$ One of the most useful tools in thyroid nodule assessment is FNA biopsy. The large-scale use of FNA in preoperative nodule evaluation has resulted in an increase in malignant specimens revealed after thyroidectomies by more than $50 \%$. At the same time, the number of patients with thyroid nodules who are referred for surgery after FNA has decreased by $50 \%$. Based on the American Thyroid Association guidelines from 2008 and Polish guidelines from 2010, the gold-standard preoperative evaluation of thyroid nodules includes ultrasonography and FNA, preceded by a thorough consideration of indications and contraindications. ${ }^{9,14,15}$ FNA is favored for its cost-effectiveness, safety in outpatient clinical conditions, ease of repetition, and high degree of specificity and sensitivity ${ }^{16}$ An important contribution to the quality improvement of FNA was made by the recommendation of 
the Bethesda System for Reporting Thyroid Cytopathology in 2008. The Bethesda classification divides the outdated descriptive FNA results into six categories: 1) nondiagnostic or unsatisfactory, 2) benign, 3) atypia of undetermined significance or follicular lesion of undetermined significance, 4) follicular neoplasm or suspicion of a follicular neoplasm, 5) suspicion of malignancy, and 6) malignant. Each category is assigned to a particular risk for malignancy, which makes the Bethesda system a valuable aid in choosing the proper treatment option for the patient, such as further nodule observation at a 6-18-48 months follow-up visit, referral for surgery, or other types of treatment., 3,17,18

Numerous studies have confirmed a high level of FNA specificity $(86 \%-100 \%)$ and sensitivity (93\%-100\%), accompanied by a low rate of false-negative results $(3 \%-6 \%)$. The exclusion of nondiagnostic and suspicious specimens improves the specificity and sensitivity in terms of malignancy over $90 \%{ }^{15,19,20}$ However, many single-center studies based on their own clinical data have shown a disturbing discrepancy between FNA and postoperative thyroid histopathology results. The degree of sensitivity and specificity obtained using FNA was much lower than expected. The rate of suspicious/nondiagnostic and false-negative FNA biopsies was also surprisingly high. The true incidence of TC revealed in patients with an undetermined FNA biopsy can vary from $7 \%$ to $48 \% .{ }^{11,13}$ Nondiagnostic/unsatisfactory (Bethesda 1) FNA biopsies are found in 2\%-20\% cases, whereas the desired rate should be below $10 \%$. In these single-center studies, the Bethesda 1 FNA biopsies rate reached up to $30 \% .{ }^{20}$ According to the Bethesda System for Reporting Thyroid Cytopathology, such results require repetition of the nodule biopsy.

The significant discrepancy in acquired false-negative FNA biopsies $(6.7 \%-26.3 \%)$ is another important issue. ${ }^{9,10,15}$ Several attempts have been made to identify the reasons for such low-quality results and to improve the clinical efficacy of FNA. Luo et $\mathrm{al}^{8}$ reported increased rates of false-negative biopsies in nodules exceeding $40 \mathrm{~mm}$ and in multinodular thyroid goiters (MNG). In patients with multiple thyroid nodules, the choice of most suspicious foci for biopsy remains a difficult task. FNA for every single nodule revealed in a multinodular case of the disease is neither practiced nor likely to be accepted by patients. When a decision to undertake further observation of diagnosed nodules is made, the later follow-up evaluation by FNA of patients with MNG can be inadequate. The authors of this study point out that less than $37 \%$ of TCs confirmed in postoperative histopathology were found in the dominant nodule. That said, nearly two-thirds of evaluated nodules remained "benign" in the preoperative biopsy, although these patients were diagnosed with cancer on the final histopathology. Choosing nodules for FNA evaluation (especially in MNG) based on size-only criteria can be misleading, since patients classified as "benign" on the basis of the thyroid biopsy can be denied surgery or require reoperation. Wang et al present the differences between false-negative rates of FNA performed in academic ( $2 \%$ ) versus community centers $(10 \%){ }^{21}$ The insufficient use of the Bethesda system for FNA description can be another issue, which was also observed in our study. ${ }^{15}$

Having analyzed the outcomes of our study, we found that the overall occurrence of TC, as well as the sex and age of patients related to particular histological TC types corresponded well with the results presented by other centers. The previously discussed tendency of a steady increase in morbidity due to papillary $\mathrm{TC}$ or rather in its detection rate due to easy-access nodule evaluation was also observed in our study. However, the disturbingly high rate $(48 \%)$ of false-negative FNA biopsies remains a serious issue. Despite the fact that a full evaluation of an FNA biopsy requires complete analysis of all FNA biopsies from patients referred for thyroid surgery in our department (2,133 patients), the number of "benign" biopsies in our patients diagnosed with cancer is unacceptable. In reference to the study reported by Luo et al, our two patients required a second operation due to the uncertainty of the surgical margins on account of contralateral localization of the cancer to that which was expected according to FNA and ultrasonography prior to surgery. ${ }^{8}$ A second surgical approach after a partial thyroidectomy is always a serious threat to patient safety because of the high risk of laryngeal nerve injury caused due to the changed anatomy of the thyroid-adjacent tissue. 3,22,23

It is of great importance that each referral for surgery should be based on an individual preoperative evaluation. A decision about the adequate range of the operation requires a thorough analysis of clinical data, such as the patient's complaints, age, sex, family history, physical examination, laboratory tests (serum calcitonin level when there is a suspicion of medullary cancer, etc), ultrasound, and FNA evaluation. It is essential that each step of the thyroid disease assessment is focused on oncological awareness. Although FNA biopsy is the gold-standard, obligatory diagnostic tool for thyroid nodule evaluation, it is still insufficient as a standalone procedure in terms of taking a decision about thyroid surgery. ${ }^{8,15,23}$ In our opinion, the unsatisfactory results of FNA utilization noted in our study fully justify the above statement. A very cautious approach toward FNA interpretation is the basic principle in our surgery department. In fact, we tend to extend the range 
of thyroid surgery, regardless of the available clinical data. In the last few years, the vast majority of thyroid surgeries performed in our department include total or nearly total thyroidectomies. Based on our own experience, we also agree that an initial total thyroidectomy is a cost-effective and safe single-step procedure. At the moment, it is the most strongly favored type of thyroid surgery in our department. The advantage of a total thyroidectomy over partial excision seems to be the most reasonable and promising choice for each patient with TC referred for surgery. 3,22

\section{Conclusion}

It is of great importance that the quality and quantity of FNA biopsies that are performed have been improved, especially due to the wide adoption of the Bethesda system. FNA remains an essential part of the process of diagnosing thyroid disease; yet, it is insufficient if it is the only decisive procedure in terms of referral for surgery. In our opinion, an initial total thyroidectomy is a safe, single-step procedure of significant value, especially in patients with TC which is not discovered during the preoperative assessment.

\section{Acknowledgment}

The authors received no financial support for this research and/or the authorship of this article.

\section{Disclosure}

The authors report no conflicts of interest in this work.

\section{References}

1. Kim TY, Kim WG, Kim WB, Shong YK. Current status and future perspectives in differentiated thyroid cancer. Endocrinol Metab (Seoul). 2014;29(3):217-225.

2. Jarzab B. Thyroid cancer. In: Gajewski P, editor. Szczeklik's Manual of Internal Medicine. Medycyna Praktyczna: Krakow; 2015.

3. Nguyen QT, Lee EJ, Huang MG, Park JI, Khullar A, Plodkowski RA. Diagnosis and treatment of patients with thyroid cancer. Am Health Drug Benefits. 2015;8(1):30-40.

4. Trigo JM, Capdevila J, Grande E, Grau J, Lianes P; Spanish Society for Medical Oncology. Thyroid cancer: SEOM clinical guidelines. Clin Transl Oncol. 2014;16(12):1035-1042.

5. Schneider DF, Chen H. New developments in the diagnosis and treatment of thyroid cancer. CA Cancer J Clin. 2013;63(6):373-394.

6. Jarzab B, Sporny S, Lange D, et al. Diagnosis and treatment of thyroid cancer-Polish guidelines. Endokrynol Pol. 2010;61(5):518-568.

OncoTargets and Therapy

\section{Publish your work in this journal}

OncoTargets and Therapy is an international, peer-reviewed, open access journal focusing on the pathological basis of all cancers, potential targets for therapy and treatment protocols employed to improve the management of cancer patients. The journal also focuses on the impact of management programs and new therapeutic agents and protocols on
7. Cibas ES, Ali SZ. NCI Thyroid FNA State of the Science Conference. The Bethesda system for reporting thyroid cytopathology. Am J Clin Pathol. 2009;132(5):658-665.

8. Luo J, McManus C, Chen H, Sippel RS. Are there predictors of malignancy in patients with multinodular goiter? J Surg Res. 2012; 174(2):207-210

9. Schiro AJ, Pinchot SN, Chen H, Sippel RS. Clinical efficacy of fineneedle aspiration biopsy of thyroid nodules in males. J Surg Res. 2010; 159(2):645-650.

10. Gupta M, Gupta S, Gupta VB. Correlation of fine needle aspiration cytology with histopathology in the diagnosis of solitary thyroid nodule. J Thyroid Res. 2010;2010:379051.

11. Morrison SA, Suh H, Hodin RA. The surgical management of thyroid cancer. Rambam Maimonides Med J. 2014;5(2):e0008.

12. Slijepcevic N, Zivaljevic V, Marinkovic J, Sipetic S, Diklic A, Paunivic I. Retrospective evaluation of the incidental finding of 403 papillary thyroid microcarcinomas in 2466 patients undergoing thyroid surgery for presumed benign thyroid disease. BMC Cancer. 2015;15:330.

13. Xing M, Haugen BR, Schlumberger M. Progress in molecular-based management of differentiated thyroid cancer. Lancet. 2013;381(9871): 1058-1069.

14. Renuka IV, Saila Bala G, Aparna C, Kumari R, Samalatha K. The Bethesda system for reporting thyroid cytopathology: interpretation and guidelines in surgical treatment. Indian J Otolaryngol Head Neck Surg. 2012;64(4):305-311.

15. Le AR, Thompson GW, Hoyt BJ. Thyroid fine-needle aspiration biopsy: an evaluation of its utility in a community setting. J Otolaryngol Head Neck Surg. 2015;44:12.

16. Bhasin TS, Mannan R, Manjari M, et al. Reproducibility of 'The Bethesda System for reporting Thyroid Cytopathology': a multicenter study with review of the literature. J Clin Diagn Res. 2013;7(6):1051-1054.

17. Negro R. What happens in a 5-year follow-up of benign thyroid nodules. J Thyroid Res. 2014;2014:459791.

18. Nou E, Kwong N, Alexander LK, Cibas ES, Margusee E, Alexander EK. Determination of the optimal time interval for repeat evaluation after a benign thyroid nodule aspiration. $J$ Clin Endocrinol Metab. 2014;99(2):510-516.

19. Rossing M, Nygaard B, Nielsen FC, Bennedbaek FN. High prevalence of papillary thyroid microcarcinoma in danish patients: a prospective study of 854 consecutive patients with a cold thyroid nodule undergoing fine-needle aspiration. Eur Thyroid J. 2012;1(2):110-117.

20. Eilers SG, LaPolice P, Mukunyadzi P, et al. Thyroid fine-needle aspiration cytology: performance data of neoplastic and malignant cases as identified from 1558 responses in the ASCP Non-GYN assessment program thyroid fine-needle performance data. Cancer Cytopathol. 2014;122(10):745-750.

21. Wang CC, Friedman L, Kennedy GC, et al. A large multicenter correlation study of thyroid nodule cytopathology and histopathology. Thyroid. 2011;21(3):243-251.

22. Vassiliou I, Tympa A, Arkadopoulos N, Nikolakopoulos F, Petropoulou T, Smyrniotis V. Total thyroidectomy as the single surgical option for benign and malignant thyroid disease: a surgical challenge. Arch Med Sci. 2013;9(1):74-78.

23. Ahmed SR, Ball DW. Incidentally discovered medullary thyroid cancer: diagnostic strategies and treatment. J Clin Endocrinol Metab. 2011; 96(5):1237-1245

\section{Dovepress}

patient perspectives such as quality of life, adherence and satisfaction. The manuscript management system is completely online and includes a very quick and fair peer-review system, which is all easy to use. Visit http://www.dovepress.com/testimonials.php to read real quotes from published authors. 